Giuseppe Lippi*, Camilla Mattiuzzi and Brandon M. Henry

\title{
Are sniffer dogs a reliable approach for diagnosing SARS-CoV-2 infection?
}

https://doi.org/10.1515/dx-2021-0034

Received March 10, 2021; accepted April 9, 2021.

published online April 20, 2021

\section{Abstract}

Objectives: Despite inter-individual variations in their diagnostic efficiency, dogs have been trained to investigate many human pathologies, especially cancer, diabetes, migraine, seizures and even infectious diseases. To this end, we performed a critical review and pooled analysis of current scientific literature on the performance of dogs trained for identifying severe acute respiratory syndrome coronavirus 2 (SARS-CoV-2)-positive human specimens.

Methods: We carried out an electronic search in PubMed, Scopus and Web of Science with the keywords "dog(s)" AND "sniffer" OR "scent” OR “smell” AND "SARS-CoV-2" OR “severe acute respiratory syndrome coronavirus 2 ” OR “coronavirus disease 2019” OR “COVID-19” within all fields, without date or language restrictions, to identify studies describing dogs' performance for identifying SARS-CoV-2 infected material.

Results: Three studies could be finally included in pooled analysis, totaling 17 dogs (47\% females), aged between 0.5 and 12 years. The pooled diagnostic sensitivity was 0.88 (95\% CI, 0.84-0.91; $\mathrm{I}^{2}, 85.3 \%$ ), the diagnostic specificity 0.99 (95\% CI, 0.99-0.99; I², 97.4\%), whilst the area under the summary receiver operating characteristic curve (SROC) was 0.979 (standard error, 0.003).

Conclusions: The notable performance observed in this pooled analysis would persuade us to suggest that adequately trained dogs could represent an intriguing and sustainable resource for purposes of rapid SARS-CoV2 mass screening.

*Corresponding author: Prof. Giuseppe Lippi, Section of Clinical Biochemistry, University of Verona, University Hospital of Verona, Piazzale L.A. Scuro, 10, 37134, Verona, Italy, Phone: +0039 045 8122970, Fax: +0039045 8124308, E-mail: giuseppe.lippi@univr.it. https://orcid.org/0000-0001-9523-9054

Camilla Mattiuzzi, Service of Clinical Governance, Provincial Agency for Social and Sanitary Services, Trento, Italy

Brandon M. Henry, Cardiac Intensive Care Unit, The Heart Institute, Cincinnati Children's Hospital Medical Center, Cincinnati, OH, USA
Keywords: coronavirus; COVID-19; diagnosis; dogs.

\section{Introduction}

Coronavirus disease 2019 (COVID-19), a severe and occasionally critical infectious disease sustained by severe acute respiratory syndrome coronavirus 2 (SARS-CoV-2), has already reached the dimension of a seemingly unstoppable pandemics. Although diagnostic testing, aimed at identifying subjects with either symptomatic or asymptomatic SARS-CoV-2 infections, is a mainstay of all prevention or containment strategies [1], the current capacity of clinical laboratories to perform the reference molecular testing on nasopharyngeal swab specimens is overwhelmed in numerous countries due to the large number of samples that would need to be tested [2]. The use of surrogate approaches, such as rapid antigen tests, is plagued by a suboptimal diagnostic sensitivity [3], whilst the promising results obtained via saliva testing [4] have still not been acknowledged by many driving scientific organizations, first among which the World Health Organization (WHO), which still only endorses the usage of nasopharyngeal or lower respiratory tract materials [5].

An incomparable olfactory sense, orders of magnitude more sensitive than in humans, is a highly specific trait characterizing almost all healthy dogs [6]. Despite inter-individual variations in diagnostic efficiency, dogs have now been trained to screen, or even detect, a vast array of human pathologies, including cancer, diabetes, migraine, seizures [7] and, quite recently, infectious diseases $[8,9]$. This has led the way to explore the possibility that dogs could be used to identify SARS-CoV-2 positive biological material, thus amplifying the armamentarium for COVID-19 screening. To this end, this study was aimed to carry out a critical review and pooled analysis of the current scientific literature on the performance of dogs trained to identify SARS-CoV-2-positive human samples.

\section{Materials and methods}

We carried out an electronic search in PubMed, Scopus and Web of Science with the keywords "dog(s)" AND "sniffer" OR "scent" OR "smell" AND "SARS-CoV-2" OR "severe acute respiratory syndrome 
coronavirus 2" OR “coronavirus disease 2019” OR “COVID-19” within all fields, without date or language restrictions (i.e., up to March 30, 2021). Two authors (G.L. and C.M.) analyzed the title, abstract and full text (when available) of all items first identified using these search criteria, selecting studies which described the diagnostic performance of dogs in identifying SARS-CoV-2 infected material (either biological or not). The references of all selected articles were also analyzed with the purpose of identifying other possibly useful documents. When available the false positive (FP), false negative (FN), true positive (TP) and true negative (TN) values were included in a pooled analysis, aimed at estimating diagnostic sensitivity, specificity and accuracy (summary receiver operating characteristic curve; SROC), with $95 \%$ confidence interval ( $95 \%$ CI). When multiple experiments were presented, the results of the last trial were included in the final pooled analysis. Between-study heterogeneity was calculated using $\chi^{2}$-test and $\mathrm{I}^{2}$ statistic. The statistical analysis was performed using Meta-DiSc 1.4 (Unit of Clinical Biostatistics team of the Ramón y Cajal Hospital, Madrid, Spain) [10]. The study was conducted in agreement with the Declaration of Helsinki and within the terms of local legislation.

\section{Results}

The electronic search led to identify 17 documents following elimination of duplicates across scientific databases. Thirteen of these items were excluded as they did not show information on dog performance for diagnosing SARS-CoV-2 infection $(\mathrm{n}=6)$, were review articles $(\mathrm{n}=4)$, editorial material $(\mathrm{n}=2)$ or correspondence/commentaries $(\mathrm{n}=1)$. No disagreement emerged between the two reviewers. Among the remaining four studies, that of Grandjean et al. was omitted due to the lack of retrievable information for calculating the diagnostic specificity [11]. Therefore, three studies could be finally included in our pooled analysis, totaling $17 \mathrm{dogs}$ ( $47 \%$ females), aged between 0.5 and 12 years, as summarized in Table 1 [12-14]. The biological material presented to the dogs for SARS-CoV-2 detection was rather heterogeneous, including nasopharyngeal swabs, saliva, sputum, nasopharyngeal or tracheal aspirates, axillary sweat samples, clothes and masks worn by humans. Molecular testing was always used as reference technique for defining SARS-CoV-2 positivity. The main results of our analysis are shown in Figure 1. The pooled diagnostic sensitivity was 0.88 (95\% CI, 0.84-0.91; $\mathrm{I}^{2}, 85.3 \%$ ), the diagnostic specificity 0.99 (95\% CI, 0.99-0.99; $\left.\mathrm{I}^{2}, 97.4 \%\right)$, whilst the area under the SROC was 0.979 (standard error, 0.003) (Figure 1).

\section{Discussion}

The results of this pooled analysis of the current scientific literature reveals that, like in many other diseased states, the rather impeccable sense of smell of dogs may represent an intriguing opportunity for the purposes of screening and/or diagnosing SARS-CoV-2 infections. The three studies included in our analysis yielded a good sensitivity (i.e., 0.88), compounded by almost perfect specificity (i.e., 0.99), overall reflected in 0.98 (SROC) diagnostic accuracy. Notably, the value of diagnostic sensitivity was found to be even higher than that calculated by Mohammadi et al. in molecular tested nasopharyngeal swab samples of patients with symptoms onset between 0 and 7 days (0.80; 95\% CI, 0.66-0.91) [15], and almost comparable to that calculated by Butler-Laporte et al. in another more recent meta-analysis (0.85; 95\% CI, 0.77-0.92) [16]. Sniffer dogs also exhibited identical specificity than that calculated in molecular tested nasopharyngeal samples by Butler-Laporte et al. (0.99; 95\% CI, 0.97-1.00) [16].

Besides the three studies included in this pooled analysis, a special mention should be made of the study by Grandjean et al. [11], which was excluded for lacking retrievable information for calculating the diagnostic specificity. This study involved six detection dogs (aged 1.3-8 years, 50\% females), who were trained to identify SARS-CoV-2 in axillary sweat samples. The success rate per dog varied between 76 and $100 \%$, displaying a cumulative value of diagnostic sensitivity of 0.92 (95\% CI, 0.88-95), which is hence well aligned with that calculated in our pooled analysis.

That said, it is undeniable that the use of dogs trained at detecting SARS-CoV-2 in human samples would have some notable advantages, but also some important drawbacks [17]. With respect to benefits, it is noteworthy to mention here that the overall costs per analysis of using a dog would be unquestionably lower than using standard techniques such as molecular or antigen testing, while enabling rapid mass screening of a large number of subjects daily, without further stressing clinical laboratories, limited personnel resources, and material and kit shortages.

With respect to the possible drawbacks, the first include the costs and the long time necessary for appropriate training, which would need to be compounded in larger cost-effectiveness investigations. Dogs are not instruments, and therefore their performance would be subjective, varying widely depending on their breed, age, individual skills, type and duration of training, as well as on the amount and type of infected material that is smelt. A dog's performance may also be impaired by tiredness, lethargy, hunger and other external distractions. It has also remained rather unclear to-date as to what are the specific volatile organic compounds (VOCs) patterns produced by the infected cells, that these friendly animals would be capable to identify in biological materials of people with SARS-CoV-2 infection [18], and how this would relate to viral load and transmissibility. To this end, a clinical trial has recently revealed that an electronic nose used for distinguishing COVID-19 positive from negative subjects by means of VOCs analysis 
Table 1: Summary of studies which explored the performance of dogs trained to identify severe acute respiratory syndrome coronavirus 2 (SARS-CoV-2) in human samples.

\begin{tabular}{|c|c|c|c|c|c|c|c|c|}
\hline Authors & $\begin{array}{r}\text { Dogs, } \\
n\end{array}$ & $\begin{array}{r}\text { Age, } \\
\text { years }\end{array}$ & Females & Attempts, $n$ & Human samples & $\begin{array}{l}\text { Reference } \\
\text { technique }\end{array}$ & Blinding & Randomization \\
\hline $\begin{array}{l}\text { Eskandari } \\
\text { et al. [12] }\end{array}$ & 3 & $1-3$ & $1(33 \%)$ & $\begin{array}{l}1,012 \\
\text { (42\% positive) }\end{array}$ & Clothes and masks & NAAT & $\begin{array}{l}\text { Yes } \\
\text { (single) }\end{array}$ & Not reported \\
\hline $\begin{array}{l}\text { Jendrny et al. } \\
\text { [13] }\end{array}$ & 8 & $0.5-12$ & $3(37 \%)$ & $\begin{array}{l}120 \\
(19 \% \text { positive) }\end{array}$ & $\begin{array}{l}\text { Saliva or tracheobronchial } \\
\text { secretions }\end{array}$ & NAAT & $\begin{array}{l}\text { Yes } \\
\text { (double) }\end{array}$ & Yes \\
\hline $\begin{array}{l}\text { Vesga et al. } \\
{[14]}\end{array}$ & 6 & $0.5-3$ & $4(67 \%)$ & $\begin{array}{l}6,000 \\
\text { (2\% positive) }\end{array}$ & $\begin{array}{l}\text { Nasopharyngeal swab, tracheal } \\
\text { aspirate, nasopharyngeal aspi- } \\
\text { rate, sputum and saliva }\end{array}$ & NAAT & $\begin{array}{l}\text { Yes } \\
\text { (single) }\end{array}$ & Yes \\
\hline
\end{tabular}

NAAT, nucleic acid amplification test.
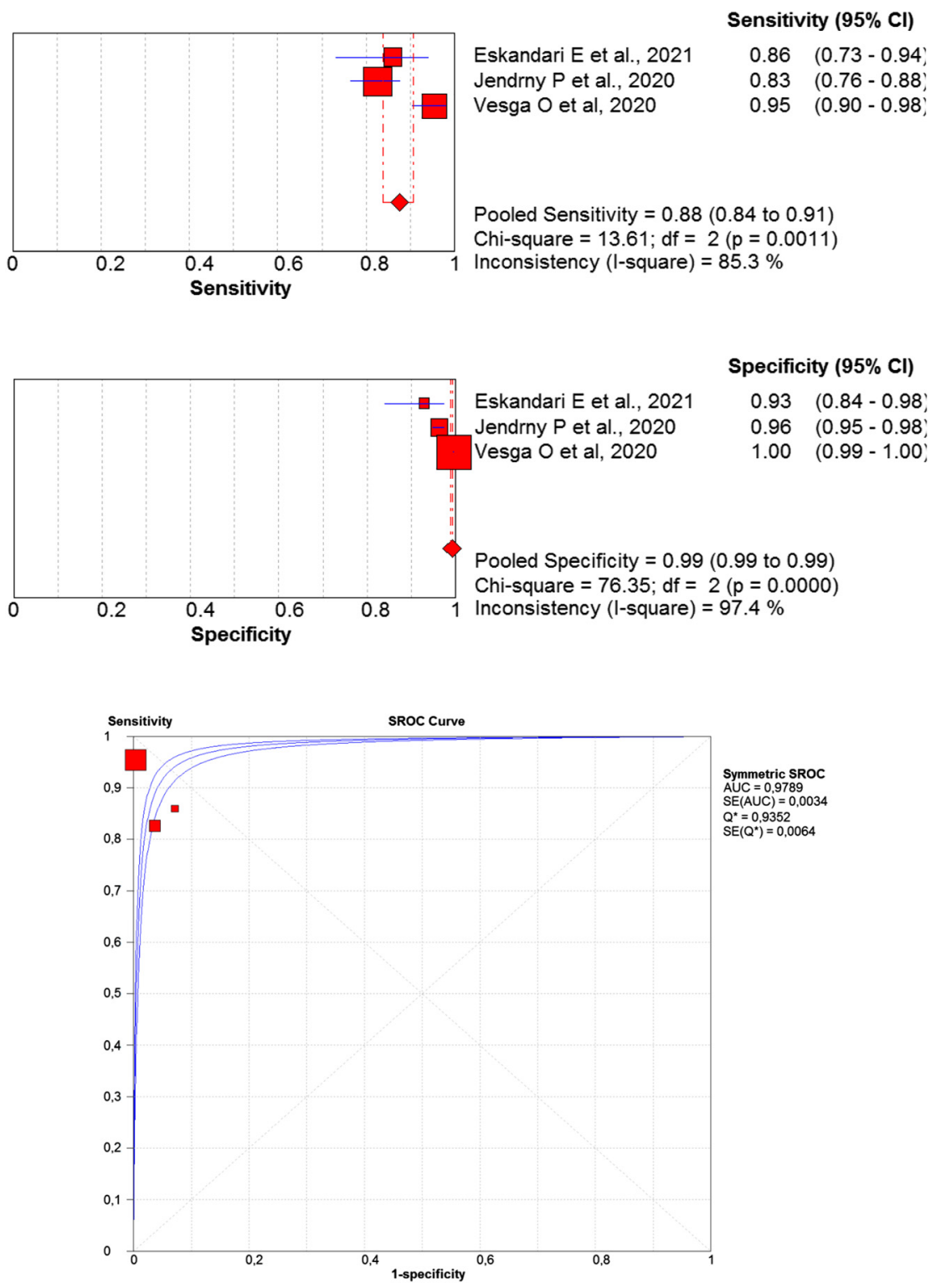

Figure 1: Pooled diagnostic sensitivity, specificity and accuracy (summary receiver operating characteristic curve; SROC) of dogs trained to identify severe acute respiratory syndrome coronavirus 2 (SARS-CoV-2) in human samples. 
displayed a value of diagnostic sensitivity that was almost indistinguishable to that of the sniffer dogs (i.e., 0.86 vs. 0.88 ), though exhibiting a much lower diagnostic specificity (i.e., 0.54 vs. 0.99) [19]. It is also worthwhile mentioning here that dogs' performance may depend on sample type and quality, whereby odors may acquire specific signatures whether the biological material is presented on swabs, liquids, clothes or other materials. Moreover, demographic features such as age, sex and ethnic origin, along with the possible presence of comorbidities may contribute to modify intensity and characteristics of the odors, thus biasing animal's performance. Finally, while it appears that dogs and other household pets are susceptible to SARS-CoV-2 infection, to what extent they suffer illness and any role such animals may play in transmission, is still being investigated [20].

In conclusion, the remarkable performance that dogs seemingly display for detecting SARS-CoV-2 infections would persuaded us to suggest that adequately trained dogs could represent an intriguing and sustainable resource for purposes of rapid COVID-19 mass screening, for example before entering crowded public places such hospitals, nursing homes, schools, airports or public transport stations, as well as concerts, sporting events and other gatherings [21]. Positive results could then be confirmed with rapid antigen or molecular testing. With the pandemic unlikely to abate anytime soon, and with the obvious awareness that molecular testing still represents the mainstay for diagnosing SARS-CoV-2 infection, the use of dogs as a screening tool is indeed worthwhile exploring further.

Research funding: None declared.

Author contributions: All authors have accepted responsibility for the entire content of this manuscript and approved its submission.

Competing interests: Authors state no conflict of interest. Informed consent: Not applicable.

Ethical approval: Not applicable.

\section{References}

1. Lippi G, Plebani $M$. The critical role of laboratory medicine during coronavirus disease 2019 (COVID-19) and other viral outbreaks. Clin Chem Lab Med 2020;58:1063-9.

2. Bohn MK, Mancini N, Loh TP, Wang CB, Grimmler M, Gramegna M, et al. IFCC interim guidelines on molecular testing of SARS-CoV-2 infection. Clin Chem Lab Med 2020;58:1993-2000.

3. Mattiuzzi C, Henry BM, Lippi G. Making sense of rapid antigen testing in severe acute respiratory syndrome coronavirus 2 (SARS-CoV-2) diagnostics. Diagnosis 2020:dx-2020-0131. https://doi.org/10.1515/dx-2020-0131 [Epub ahead of print].
4. Basso D, Aita A, Padoan A, Cosma C, Navaglia F, Moz S, et al. Salivary SARS-CoV-2 antigen rapid detection: a prospective cohort study. Clin Chim Acta 2021;517:54-9.

5. World Health Organization. Diagnostic testing for SARS-CoV-2 interim guidance. Geneva, Switzerland: World Health Organization; 2020.

6. Lippi G, Plebani M. Diabetes alert dogs: a narrative critical overview. Clin Chem Lab Med 2019;57:452-8.

7. Lippi G, Cervellin G. Canine olfactory detection of cancer versus laboratory testing: myth or opportunity? Clin Chem Lab Med 2012; 50:435-9.

8. Angle TC, Passler T, Waggoner PL, Fischer TD, Rogers B, Galik PK, et al. Real-time detection of a virus using detection dogs. Front Vet Sci 2016;2:79.

9. Cambau E, Poljak M. Sniffing animals as a diagnostic tool in infectious diseases. Clin Microbiol Infect 2020;26:431-5.

10. Zamora J, Abraira V, Muriel A, Khan KS, Coomarasamy A. MetaDiSc: a software for meta-analysis of test accuracy data. BMC Med Res Methodol 2006;6:31.

11. Grandjean D, Sarkis R, Lecoq-Julien C, Benard A, Roger V, Levesque $\mathrm{E}$, et al. Can the detection dog alert on COVID-19 positive persons by sniffing axillary sweat samples? A proof-ofconcept study. PloS One 2020;15:e0243122.

12. Eskandari E, Ahmadi Marzaleh M, Roudgari H, Hamidi Farahani R, Nezami-Asl A, Laripour R, et al. Sniffer dogs as a screening/ diagnostic tool for COVID-19: a proof of concept study. BMC Infect Dis 2021;21:243.

13. Jendrny P, Schulz C, Twele F, Meller S, von Köckritz-Blickwede M, Osterhaus ADME, et al. Scent dog identification of samples from COVID-19 patients - a pilot study. BMC Infect Dis 2020;20: 536.

14. Vesga O, Valencia AF, Mira A, Ossa F, Ocampo E, Agudelo M, et al. Osorio. Dog savior: immediate scent-detection of SARS-COV-2 by trained dogs. BioRxiv 2020.06.17:158105. https://doi.org/10. 1101/2020.06.17.158105.

15. Mohammadi A, Esmaeilzadeh E, Li Y, Bosch RJ, Li JZ. SARS-CoV-2 detection in different respiratory sites: a systematic review and meta-analysis. EBioMedicine 2020;59:102903.

16. Butler-Laporte G, Lawandi A, Schiller I, Yao M, Dendukuri N, McDonald EG, et al. Comparison of saliva and nasopharyngeal swab nucleic acid amplification testing for detection of SARS-CoV-2: a systematic review and meta-analysis. JAMA Intern Med 2021;181:353-60.

17. Lippi G, Heaney LM. The "olfactory fingerprint": can diagnostics be improved by combining canine and digital noses? Clin Chem Lab Med 2020;58:958-67.

18. Sharun K, Jose B, Tiwari R, Natesan S, Dhama K. Biodetection dogs for COVID-19: an alternative diagnostic screening strategy. Publ Health 2021:S0033-3506(21)00007-X. https://doi.org/10.1016/j. puhe.2020.12.01310.1016/j.puhe.2020.12.013 [Epub ahead of print].

19. Wintjens AGWE, Hintzen KFH, Engelen SME, Lubbers T, Savelkoul PHM, Wesseling G, et al. Applying the electronic nose for pre-operative SARS-CoV-2 screening. Surg Endosc 2020:1-8. https://doi.org/10.1007/s00464-020-08169-010.1007/s00464020-08169-0 [Epub ahead of print].

20. Centers for Disease Control and Prevention. COVID-19 and animals. Available from: https://www.cdc.gov/coronavirus/2019-ncov/ daily-life-coping/animals.html [Accessed 11 Mar 2021].

21. Dickey $\mathrm{T}$, Junqueira $\mathrm{H}$. Toward the use of medical scent detection dogs for COVID-19 screening. J Am Osteopath Assoc 2021;121:141-8. 